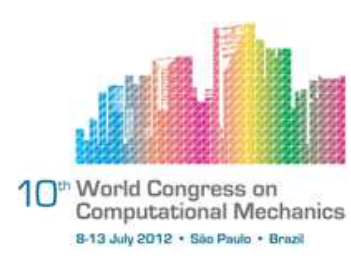

\title{
CONTACT BETWEEN ROUGH SURFACES : CRYSTAL PLASTICITY INFLUENCE ON THE CONTACT TIGHTNESS ESTIMATION
}

\author{
J. Durand ${ }^{1}$, H. Proudhon ${ }^{1}$, G. Cailletaud ${ }^{1}$ \\ ${ }^{1}$ Department of Comportment et Calcul des Structures, Centre des matériaux MINES Paris- \\ Tech (\{julian.durand, henry.proudhon, georges.cailletaud $\} @$ mines-paristech.fr)
}

\begin{abstract}
This paper is devoted to analyse the influence of material behaviour in a normal frictionless contact between a rough surface and a rigid plane. By means of Finite Element code Zset/ZéBuLoN, crystal plasticity models are determined and compared to a von Mises elastoplastic behaviour. The influence of grain orientation but also elastic anisotropy are put forward. Stress distributions, cumulated plastic strain, global responses and fluid flow in the resulting aperture field between surfaces are then quantified and compared.
\end{abstract}

Keywords : Mechanical contact, Rough surface, Crystal plasticity, Fluid flow.

\section{INTRODUCTION}

Roughness is caracteristic of every surface and has important consequences in various and numerous applications. From thermal and electrical conductivity to wear processes, rough surfaces in contact are now widely studied. The contact between rough surfaces is in fact a one to one contact between many asperities, depending on the roughness of the contact pairs. This represents a strong deviation from the perfect contact assumed in the Hertz theory, the real contact area being usually significantly smaller than the apparent contact area, and proportional to the normal load. The presence of free volume between surface also arouses research interests, notably for tightness contact and percolation studies, and has been developed by many authors [1, 2, 3]. Since Abbot and Firestone [4], a rough surface characterisation is made by means of vertical and horizontal parameters [5]. The investigation of the mechanical contact generally follows two types of approach, either stochastic or deterministic. The first one was introduced by Greenwood and Williamson [6] in considering elastic and spherical asperities with a constant radius of curvature. These kind of approaches have had a considerable impact on contact studies, due to the quick and rather good solution they provide, and were developed in a lot of works [7, 8, 9, 10]. Deterministic approaches were developed to propose a more realistic geometrical description. Asperities are then represented by means of mathematical functions. The influence of material behaviour is studied, for elastic perfectly plastic [11] or elastoplastic [12] constitutive equations. The interaction between asperity is also integrated in some models [13, 14, 15]. Finite Element methods were also used to solve the contact problem between artificial fractal surfaces [16]. Starting from Mandelbrot's work, this 
interesting research direction allowed to demonstrate the fractal character of rough surfaces [17, 18, 19, 20, 21]. At the roughness scale (micrometer), the question on the constitutive law used in mechanical contact is questionable. The consideration of an homogeneous material may not be physical anymore. At this scale, a steel is defined by grains and crystal orientations which behave according to crystal plasticity model. The purpose of this paper is to introduce such a behaviour in our mechanical contact problem. The paper will then present, in a first part, a numerical crystal plasticity model. In a second time, the study of a single asperity in contact with a rigid plane will be done in order to apprehend differences induced by such a behaviour in comparison to macroscopic one. Finally, the asperity will be substituted by a rough surface in order to compare different behaviour from a fluid flow point of view.

\section{MATERIAL BEHAVIOURS}

This article will present numerical computation of the contact between a rigid plane and a rough surface. A comparison between several behaviour will be shown. The behaviour only concern the rough surface in contact. In that way, three constitutive models will be compared : elastoplastic model, crystal plasticity with isotropic and anisotropic elasticity. The determination of elastoplastic model will not be presented here, see previous work [22].

Due to the scale of the study (micrometer), the use of a macroscopic constitutive law is questionable. At such a scale, a steel material deformation is the result of dislocation slip caused by an applied stress. Thus, a grain behaviour is governed by a monocristal model which incorporates slip systems. These slip systems are defined by a slip plane (normal $\underline{n}^{s}$ ) and a slip direction $\underline{l}^{s}$. Then, the yield surface relies on the critical resolved shear stress, calculated from the stress tensor $\underset{\sim}{\sigma}$.

$$
\begin{gathered}
\tau^{s}=\underset{\sim}{\boldsymbol{\sigma}}: \underline{\sim}^{s} \\
{\underset{\sim}{\sim}}^{s}=\frac{1}{2}\left(\left(\underline{\mathbf{l}}^{\mathbf{s}} \otimes \underline{\mathbf{n}}^{\mathbf{s}}\right)+\left(\underline{\mathbf{n}}^{\mathbf{s}} \otimes \underline{\mathbf{l}}^{\mathbf{s}}\right)\right)
\end{gathered}
$$

where ${\underset{\sim}{\mathbf{m}}}^{s}$ represents the orientation tensor of the system $s$. In small deformation, the strain

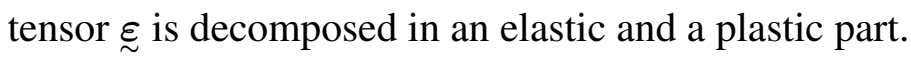

$$
\underset{\sim}{\varepsilon}={\underset{\sim}{\varepsilon}}^{e}+{\underset{\sim}{\varepsilon}}^{p}
$$

Crystal plasticity models generally assume that dislocation slip is the predominant mechanism of deformation. The average plastic strain in a grain is described as being the sum of the slip rate $\dot{\gamma}^{s}$ on each system :

$$
{\underset{\sim}{\varepsilon}}^{p}=\sum_{s} \dot{\gamma}^{s}{\underset{\sim}{\mathbf{m}}}^{s}
$$

The monocrystal model use in the present work is the Méric-Cailletaud model [23, 24]. This model suggests a phenomenological formulation of deformation process. Thus, the plastic slip rate of each slip system uses a Norton's type viscoplastic flow behaviour with an isotropic hardening term $r^{s}$ and a cinematic hardening term $x^{s}$.

$$
\dot{\gamma}^{s}=\dot{v}^{s} \operatorname{sign}\left(\tau^{s}-x^{s}\right)
$$




$$
\dot{v}^{s}=\left\langle\frac{\left|\tau^{s}-x^{s}\right|-\tau^{0}-r^{s}}{K}\right\rangle^{n} \text { with }\left\{\begin{array}{lll}
\langle x\rangle=x & \text { if } \quad x \geq 0 \\
\langle x\rangle=0 & \text { if } \quad x \leq 0
\end{array}\right.
$$

where $\tau^{0}$ is the critical resolved shear stress, $\dot{v}^{s}$ the slip velocity on the system $s, K$ and $n$ the material coefficients of the Norton's law.

This model takes into account non-linear hardening laws. The evolution of the hardening describes the increase of the critical shear stress on a system with the cumulated slip on each system. Its expression introduce an interaction matrix $h_{s r}$ :

$$
r^{s}=b Q \sum_{r} h_{s r} \rho_{r} ; \quad \text { avec } \dot{\rho}^{s}=\left(1-b \rho^{s}\right) \dot{v}^{s}
$$

The description of a kinematic hardening at the slip system scale relies on the idea to introduce an effective shear stress $\left|\tau^{s}-x^{s}\right|$ on a slip system in order to take into account interaction between dislocation at large distance. This hardening is written :

$$
x^{s}=c \alpha^{s} ; \text { with } \dot{\alpha}^{s}=\dot{\gamma}^{s}-d \alpha^{s} \dot{v}^{s}
$$

The homogenization between the monocrystal and the polycrystal can be realized by means of mean field models as Kröner model [25], Hill model [26], Berveiller-Zaoui (BZ) [27] or Cailletaud-Pilvin ( $\beta$ rule) [28]. Here, the identification of the crystal plasticity law parameters were obtained using of BZ model. In a agregate computation by finite element method, a grain is characterised only by its crystal orientation if we keep aside geometric characteristic. In that way, a phase $i$ is defined by all the grain with the same orientation. We also associate a volume fraction $f_{i}$ to each phase. Stress tensors $(\underset{\sim}{\sigma}, \underset{\sim}{\Sigma})$ and plastic strain rate $\left({\underset{\sim}{\varepsilon}}^{p},{\underset{\sim}{\mathbf{E}}}^{p}\right)$ need to be define at the local and macroscopic scales.

$$
\underset{\sim}{\Sigma}=\sum_{i} f_{i}{\underset{\sim}{\boldsymbol{\sigma}}}^{i} \text { and } \quad{\underset{\sim}{\mathbf{E}}}^{p}=\sum_{i} f_{i}{\underset{\underset{\varepsilon}{p}}{p, i}}^{p}
$$

The BZ model considers an interaction law which is written as follow :

$$
\begin{gathered}
{\underset{\sim}{\boldsymbol{\sigma}}}^{i}=\underset{\sim}{\boldsymbol{\Sigma}}+2 \mu(1-\beta) \alpha \underset{\sim}{\left(\mathbf{E}^{p}-{\underset{\sim}{\varepsilon}}^{p, i}\right)} \\
\beta=\frac{2(4-5 \nu)}{15(1-\nu)} \\
\frac{1}{\alpha}=1+\frac{3}{2} \mu \frac{{\underset{\sim}{\mathbf{E}}}^{\text {Mises }}}{{\underset{\sim}{\text { Mises }}}^{\text {Mis }}}
\end{gathered}
$$

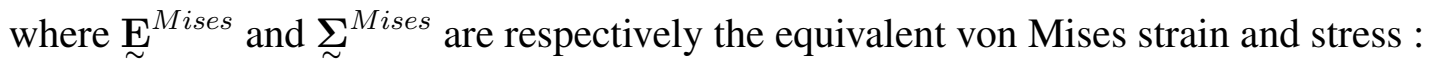

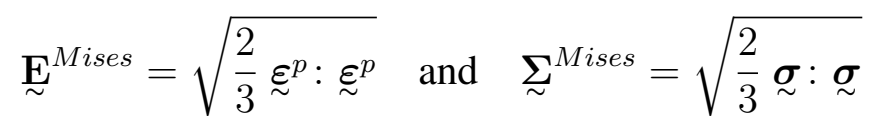

In our study, we do not give attention to the material viscosity. That is why the Norton parameters will be taken in a way to reduce this viscosity ( $K=10$ and $n=25$ ). Our material microstructure is a face centered cubic (fcc), and so, bring into play six coefficients for the 


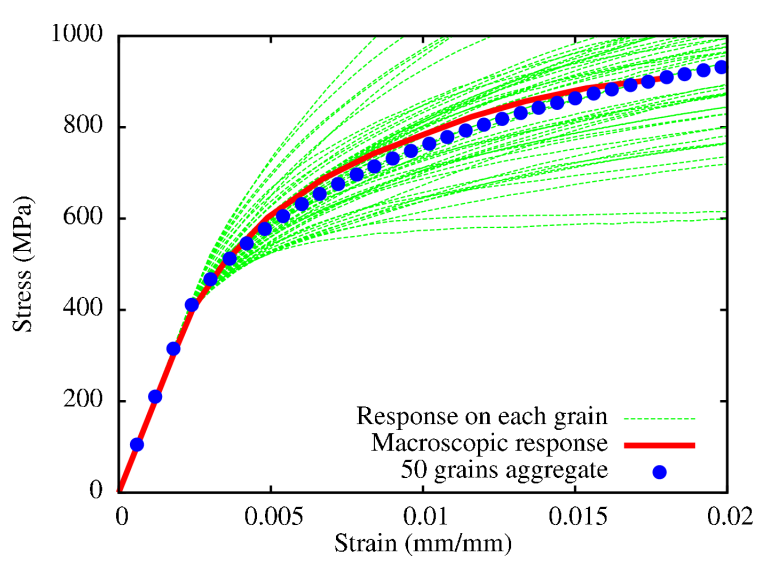

(a)

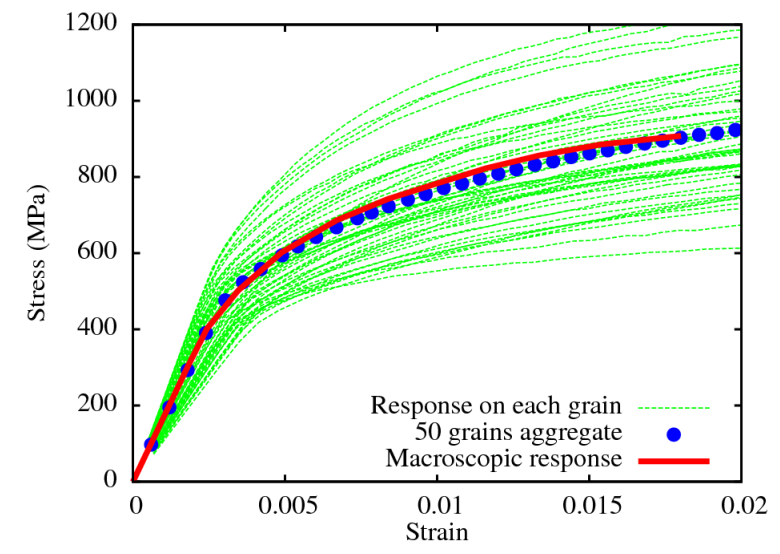

(b)

FIG. 1. grains responses (green) and whole agregate response (blue) obtained in a uniaxial tensile simulation with : a) a crystal plasticity model with an isotropic elasticity and b) a crystal plasticity behaviour with anisotropic elasticity

interaction matrix. Here, we take all these coefficients equal to 1 (Taylor hardening). By means of the finite element code Zset/ZéBuLoN the identification of the constitutive law parameters was performed by simulations on one Gauss point with forty different crystal orientations. The validation of the resulting monocrystal law was then realized on an agregate composed by 40 different grains (see figure 1 a.).

The present work will provide finite element results obtained by means of two crystal models. These models are based on the same equations which govern plastic deformation, the only difference between them is that the second one introduces an anisotropic elasticity. Therefore, the elasticity of the material is defined by means of three elastic coefficients : $y_{1111}, y_{1212}$ and $y_{1122}$. In order to determine these additional parameters, we assume here that the anisotropy of our material is similar to iron. The determination of these elastic parameters was done done thanks to a based Hill method ([29]). These parameters are then : $y_{1111}=237$ $\mathrm{GPa}, y_{1212}=141 \mathrm{GPa}$ and $y_{1122}=116 \mathrm{GPa}$. The validation of this second model need to be realized on a larger agregate (100 grains) due to its higher degree of anisotropy. Figure $1 \mathrm{~b}$. shows the responses obtained for each grain and for the whole agregate in an uniaxial traction simulation.

\section{ASPERITY IN CONTACT}

First, the study of the contact between a monocristal asperity and a rigid plane is observed. By this analysis, the objective is to see and understand the consequences brought by a crystal plasticity approach in this kind of problem. In that way, we consider here four cases (see figure 3 a.) : 1. macroscopic von Mises elastoplastic behaviour 2.-3. crystal plasticity model with isotropic elasticity 4. crystal plasticity model with anisotropic elasticity (a random orientation is affected on each monocrystal asperity case). The interest of studying anisotropic elasticity comes from the works of C. Vallet [30]. In this work, he brought forward the fact that reducing elasticity leads to a better estimation of the contact transmissivity in comparison to experimental results. The same geometry is used for all cases : asperity is axisymmetric and 
sinusoidal, has a width of $5 \mu \mathrm{m}$ and a height of $1.125 \mu \mathrm{m}$. Under the surface, the bulk material is taken sufficiently large to avoid edge effects. All the lateral faces are block in their orthogonal direction and the bottom of the asperity mesh is totally blocked. The rigid plane is moved toward the asperity. During the computation, its movement is governed by displacement. The maximum displacement $U$ reaches the value of $1 \mu \mathrm{m}$. The figures 2 a., b., c. and d. show the von Mises stress obtained on the top of the asperity at the end of the simulations. We can easily observe the loss of homogeneity induced by the crystal plasticity model : stress field is more complex in these cases. Maximal values are almost two times higher and more localised to contact zone than in the macroscopic case. Smaller stress values can also be pointed out on the asperity surface in the three monocrystal cases. The behaviour symmetry of the case 1 . also disappears when the crystal model is used. Thank to that, we are able to see the crystal orientation influence. As this orientation is different from a case to the other, the activated slip systems in the material are different. In that way, high stress zones occur at different localisations on the asperity. Concerning the cumulative plastic strain, differences between the four cases can be revealed (figures 2 e., f., g. and h.). Crystal models bring to disymmetric results. Nevertheless the gradient of plastic strain stays similar : maximal and mininal strain obtained on the asperity are identical in all cases. The orientation influence is also observable between crystal cases. High strained zones are always concentrated on the summit of the asperity but their shape do not differ as far as the von Mises stresses. Vertical displacements are visible on the figures 2 i., j., k. and 1.. For the same final displacement between the rigid plane and the asperity, the contact area appears to be similar in each study. However, the vertical displacement induced by the contact on the asperity neighbourhood presents significant differences when you compare the macroscopic approach to the crystal ones, but also between the three crystal cases. Globally, neighbourhood displacement locally reaches higher values in crystal approaches (it has to be noted that isovalues have been chosen in order to make more visible these differences). Moreover, the repartition of high displacement zones is strongly dependant on the crystal orientation chosen. The studies on plastic strain and vertical displacement allow us to affirm that the integration of a crystal plasticity model makes the deformation, and so the stress, stronger in the neighbourhood of an asperity. This kind of behaviour consequently suggests that, locally, the topology of a rough surface in contact with a rigid plane can present differences due to crystal plasticity and crystal orientation.

All the previous figures are able to provide us interesting informations on model behaviour. The orientation and the constitutive model play apparently an important role in the way an asperity has to be deformed. Another interesting result concerns the responses $F(U)$ and $A / A_{0}(F)$ (see figure 3 b. and c.), where $F$ is the applied load, $U$ the displacement between the two bodies, $A$ the real contact area and $A_{0}$ the nominal area of the asperity. Two remarks can be done. The first one is that once again a difference between crystal and macroscopic approach is present. The crystal approach need a higher applied load to reach a specific displacement between the two bodies in contact. In that sense, the asperity appears to be more rigid, plastically but also elastically speaking. The choice of the crystal orientation in case 4 . was done in order to have the lowest elasticity (figure 1 b. and 3 a.). By this case we are able to quantify the importance of elasticity constants. Indeed, the anisotropy leads to smaller values for $F(U)$ and $A / A_{0}(F)$ than other crystal approaches for a specific displacement. The second 


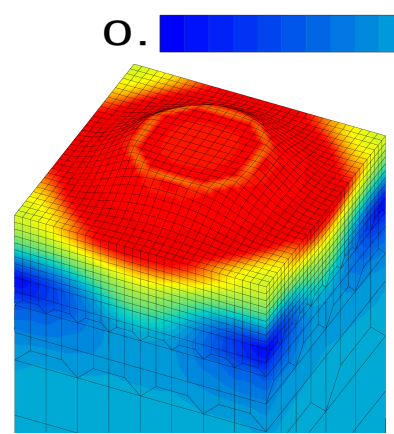

(a)

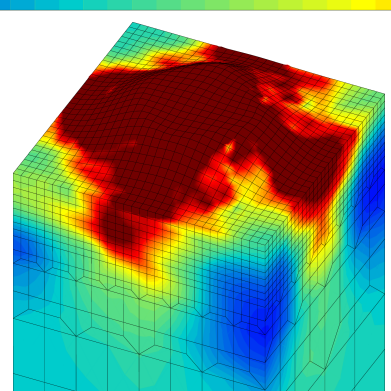

(b)

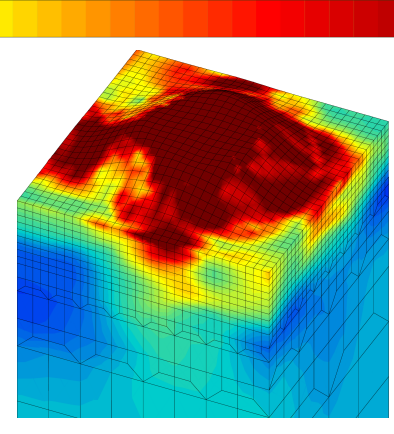

(c)

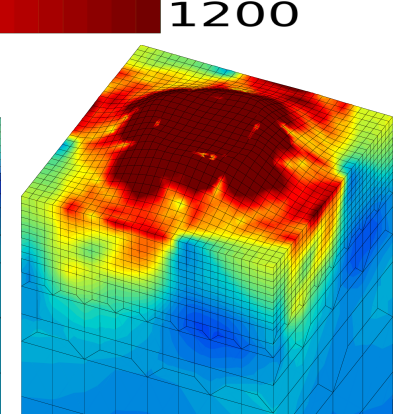

(d)

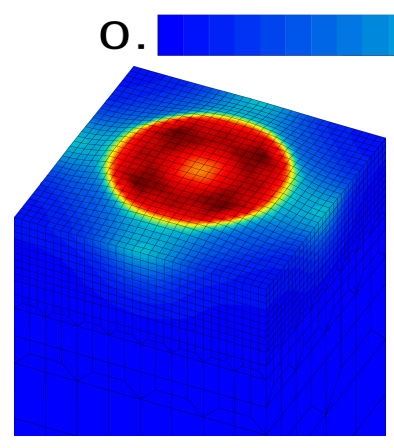

(e)

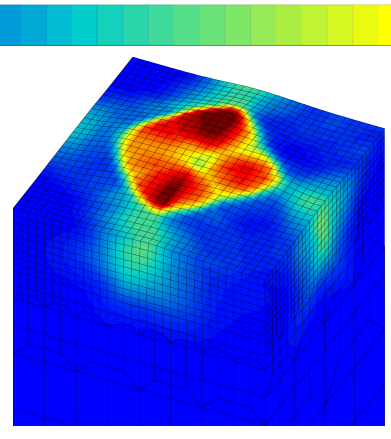

(f)

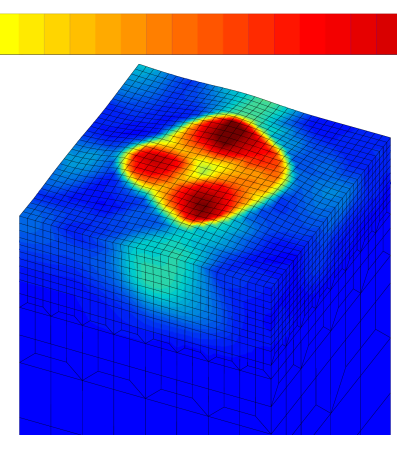

(g)

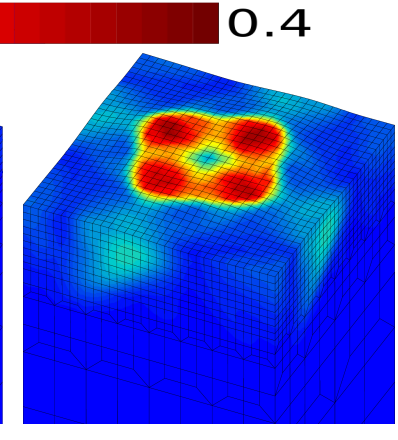

(h)

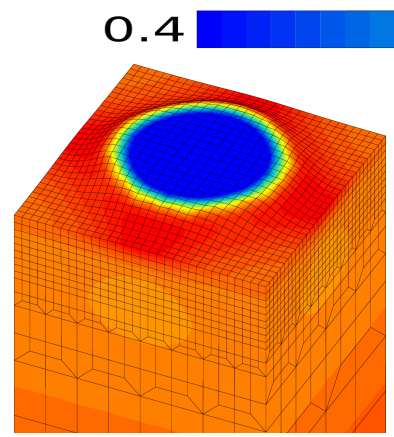

(i)

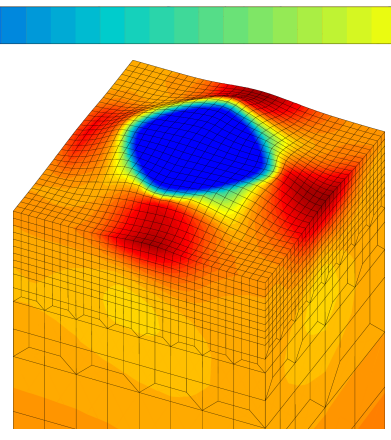

(j)

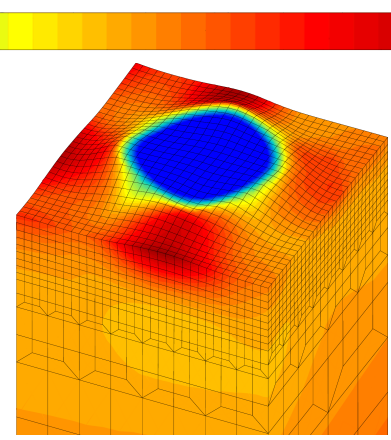

$(\mathrm{k})$
0.8

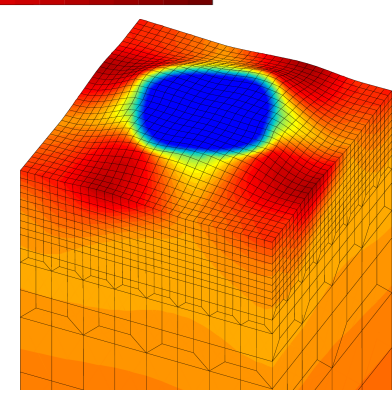

(1)

FIG. 2. Results obtained for four different asperity behaviours (one macroscopic elastoplastic, two crystal plasticity and one crystal plasticity with anisotropic elasticity) for a displacement between the rigid plane and the asperity equal to $0.6 \mu m-a), b), c)$ and d) von Mises stress distribution on the asperity surface e), f), g) and h) cumulative plastic strain on the asperity surface i), j), k) and l) vertical displacement (micrometer)

remark concerns the impact of crystal orientation. Surprisingly, the global responses of case 2. and 3. are really close to each other. In crystal plasticity approaches, the grain orientation modifies the plastic part of tensile test response. These solicitation direction is responsible of variations in the monocristal response. The activation of a slip system depends on the angle between this solicitation direction and the normal to the slip plane. That is why only few systems are actived in tensile test. The triaxiality of our problem can reach important values near the contact zone (between -1 and -2 ). This means that several solicitation directions are present in the asperity. Consequently, the activated slip systems are not necessarily the same as in a tensile test. The behaviour differences between several crystal orientation are then not as important as in this kind of test. In addition, von Mises and crystal plasticity mo- 
dels are based on the assumption of plastic incompressibility. In our study, the deformation of the rough surface is forced by the rigid plane. The mechanical conditions are really extremes (cumulative plastic strain can reach higher values than 200\%) and rub differences induced by crystal orientation.

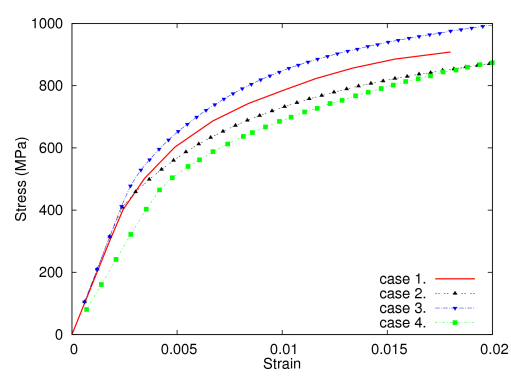

(a)

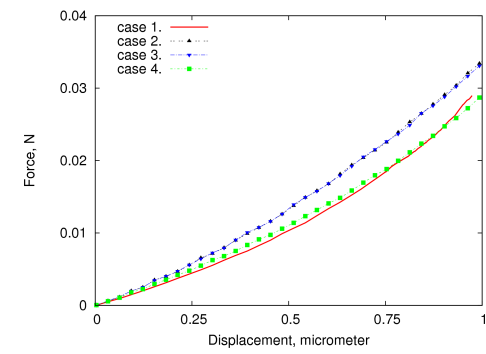

(b)

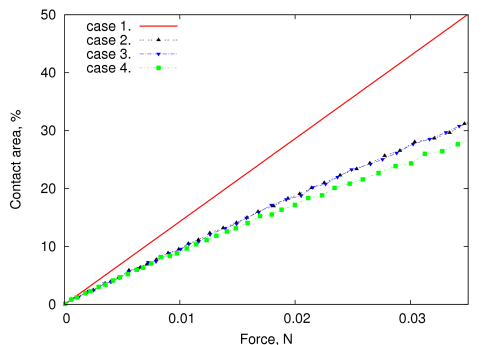

(c)

FIG. 3. a. Tensile test responses of the four models studied - b. and c. Global responses $F(U)$ and $A(F)$ of the contact between an asperity and a rigid plane

\section{ROUGH SURFACE IN CONTACT}

In a second time, rough surfaces were used for finite element analysis. Once again, four cases are developed with the same constitutive laws as in the previous part. The only difference concern crystal orientation. In this study, orientation which provides the extremes tensile test responses are chosen for case 2. and 3. : they correspond to the orientation $<111>$ and $<110>$ (see figure 4 a.). The dimension of the surface is $26 \mu \mathrm{m} \times 31 \mu \mathrm{m}$. About 80 asperities are composing the surface. Boundary conditions are taken identical as in the asperity study. The maximum displacement of the rigid plane toward the rough surface is equal to $1 \mu \mathrm{m}$. At such a displacement, the corresponding applied load is about $0.5 \mathrm{~N}$, which correspond to an apparent contact pressure on the surface equal to $600 \mathrm{MPa}$. The observation of the global response reveals the same conclusions as in the previous part (see figure $4 \mathrm{~b}$. and c.). The crystal plasticity generates higher applied load for a same displacement than in the macroscopic approach. The impact of the crystal orientation is once again not visible. The contact zones are the place of extreme stress and deformation in the three directions whereas bulk material remains in elastic regime. The anisotropy of elasticity in the case 4. provides lower responses compare to the two other crystal plasticity cases but is still higher than the case 1.. Consequently, the plasticity influence appears to be negligible while elasticity provides differences. However, the contact area does not seem to be dependant on both elasticity and plasticity. Nevertheless, the contact area shows important differences between crystal and macroscopic models.

Von Mises stress distribution is shown in the figures 5 a., b., c. and d.. Differences are not as significant as in the previous part. Of course, the distribution of this criterion on the surface remains different, especially between macroscopic and crystal cases : the high stress zones are less homogeneous and presents higher constraints in the second cases. Moreover, we can also observe differences due to elasticity anisotropy : the high stresses zones are smaller (in term of size) and regions between asperities in contact are less constrained. Nevertheless, 

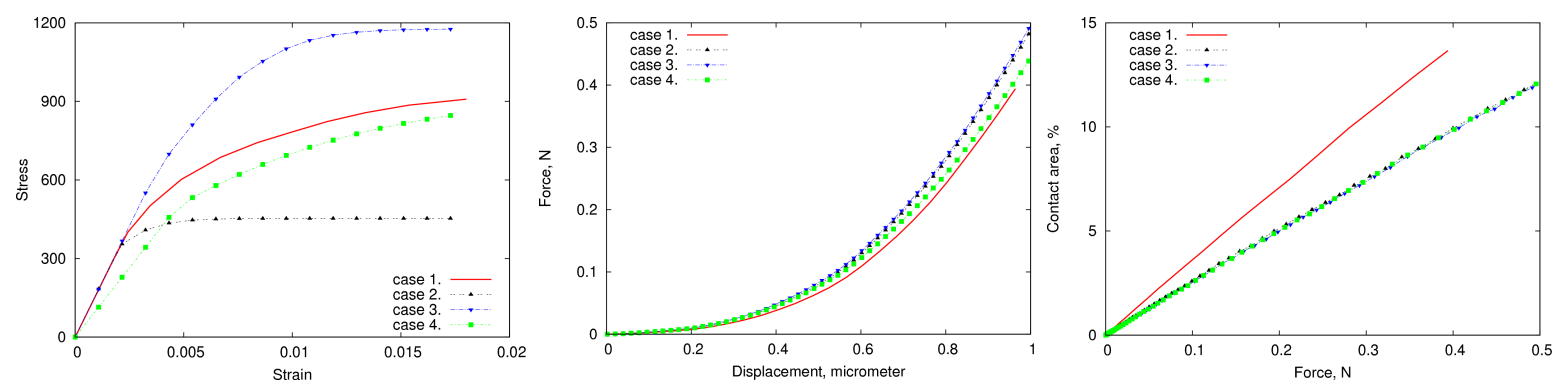

FIG. 4. a. Tensile test responses of the four models studied - b. and c. Global responses $F(U)$ and $A(F)$ of the contact between an asperity and a rigid plane

the analysis of plasticity (not shown here) reveals really few differences for all cases. The concerned zones present variations in their shape but their intensity as well as their size are almost similar. In conclusion, these results once again put forward the fact that crystal plasticity effectively brings to local differences on the rough surface deformation but does not modify global responses of this mechanical contact problem.

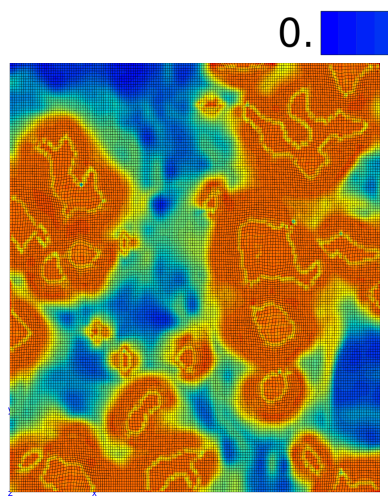

(a)

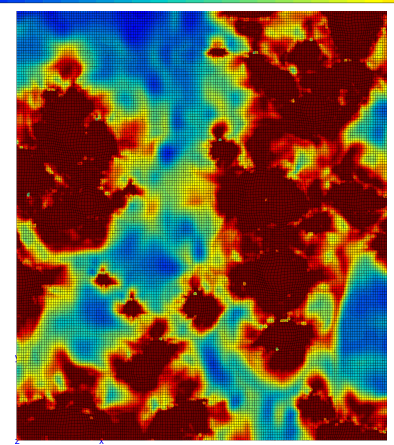

(b)

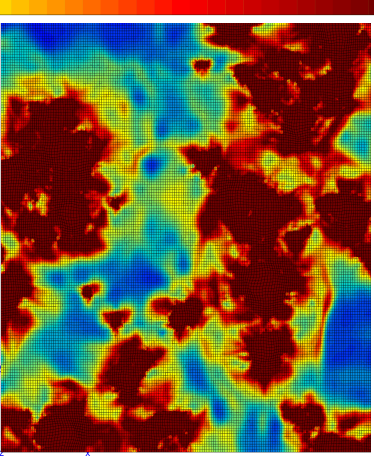

(c)

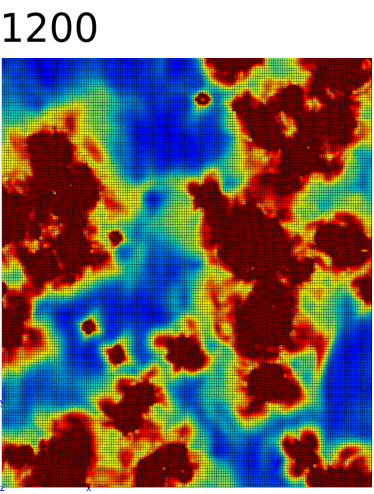

(d)

FIG. 5. Von mises stress distribution for a displacement $U=0.6 \mu \mathrm{m}: \mathrm{a}$. von Mises elastoplastic behaviour $-\mathrm{b}$. monocristal behaviour (orientation $<110>$ ) $-\mathrm{c}$. monocristal behaviour (orientation $<111>$ ) $-\mathrm{d}$. monocrystal behaviour with elastic anisotropy

The final aim of our study is to observe the influence of crystal plasticity models on the contact tightness. Several works were done in order to quantify the resulting fluid flow between a rigid plane and a rough surface [30, 1]. The fluid flow between two surfaces is strongly dependant on the aperture field between them. Figures 6 illustrate this aspect. They come from a finite element analysis of the fluid flow between the same surfaces as before. We can observe the nodal velocities of the fluid at different applied loads. A numerical model was developed in order to mesh the free volume between surfaces in contact. The fluid flow simulation were then perform by blocking all the faces with wall condition and by introducing a pressure gradient between the entrance and the exit of this fluid problem. Here, the pressure gradient is taken equal to $1 . e-6 \mathrm{MPa}$. The fluid used in the stationary simulation is ethanol, and the only parameters needed are its density $\left(8.08 \cdot 10^{-10}\right.$ tonne. $\left.\mathrm{mm}^{-3}\right)$ and its viscosity (2.73.10 ${ }^{-9}$ MPa.s).

These fluid flow simulations allow us to understand the percolation of our problem. 


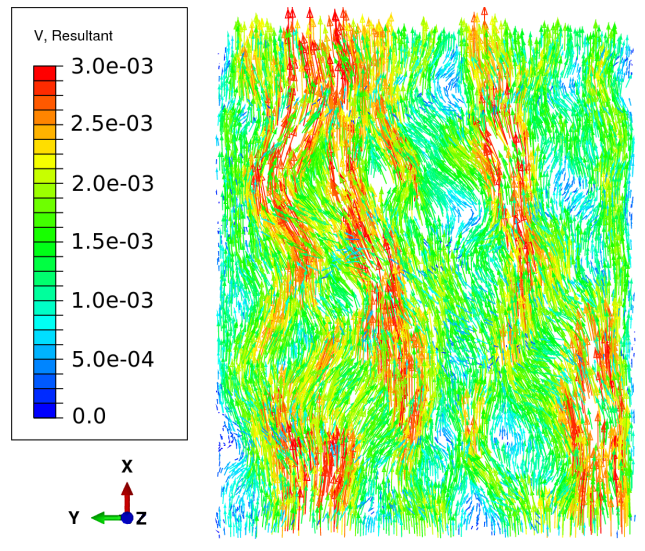

(a)

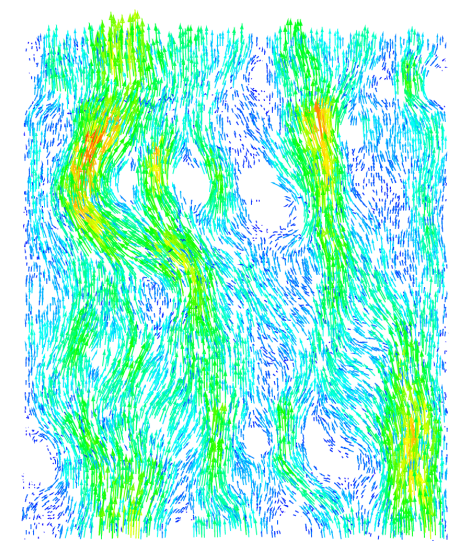

(b)

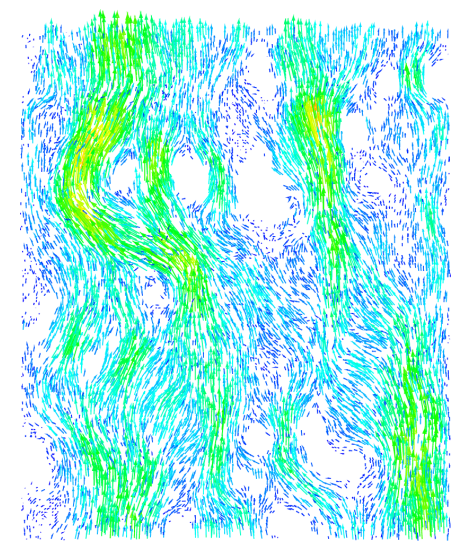

(c)

FIG. 6. Nodal velocities obtained from fluid flow simulations in the resulting free volume between a rough surface with macroscopic behaviour and a rigid plane at different displacement : a. initial position - b. $U=0.4 \mu m-$ c. $U=0.8 \mu m$ (legend is in $m m / s$ )

As the load increases, the contact zones grow, and so, the fluid is enforced to flow according to preferential tracks. The percolation is not reach during these simulation but we understand that at higher contact pressure between the rough surface and the rigid plane, the fluid will not be able to cross the surface. Thanks to these simulation the flow rate at the exit of the problem can be computed. The transmissivity $K$ of the surface can then be determined :

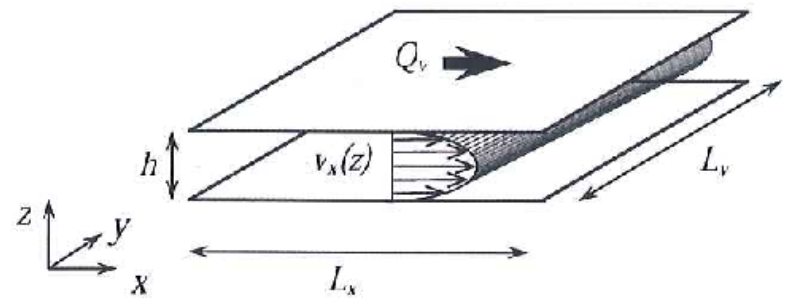

$$
\frac{Q_{v}}{L_{y}}=K \times \frac{\Delta P}{L_{x} \times \mu}
$$

where $Q_{v}$ is the flow rate in $\mathrm{X}$ direction, $\Delta P$ the pressure gradient, $\mu$ the fluid viscosity, $L_{x}$ and $L_{y}$ the dimensions of the problem.

Coupling rough surface calculations and equation 14 , the evolution of the transmissivity as a function of the applied load was plotted for each behaviour (see figure 7). As for previous studies, it is interesting to see that crystal behaviours bring to similar curves. The crystal orientation, as well as the anisotropic elasticity, do not lead to significant discrepancy. However, we can also remark that crystal models give higher transmissivity than the macroscopic one. Globally a crystal plasticity approach make the rough surface more rigid and consequently, to reach the same displacement between two bodies, the load needs to be higher in crystal cases. Thus, it appears that crystal plasticity engenders differences in the study of contact tightness. The local geometry of a rough surface is not really impacted by the behaviour but globally physical quantities differ from a behaviour to the other and generate deviation in responses of a rough surface in contact with a rigid plane. 


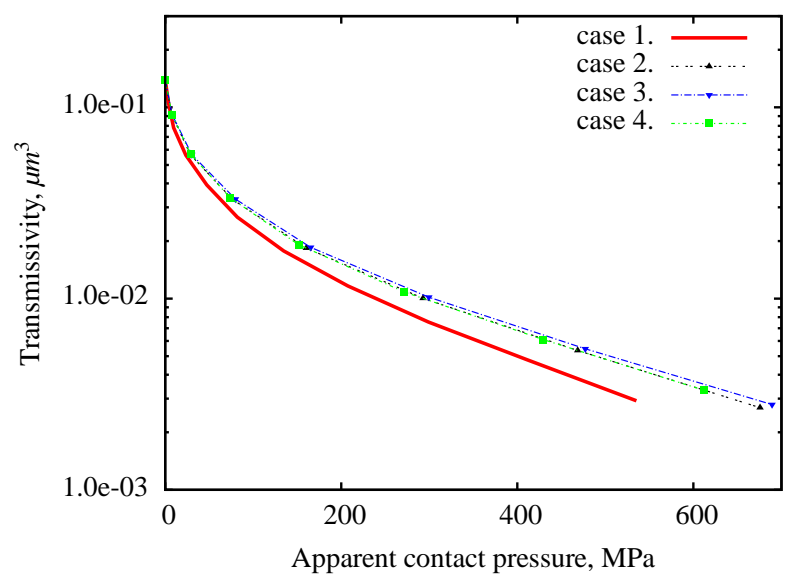

FIG. 7. Evolution of the transmissivity during the loading for each case

\section{CONCLUSION}

This paper has pointed out several results concerning the contact problem between an elastoplastic rough surface and a rigid plane. The introduction of crystal plasticity model in such a problem was carried out and compared to a macroscopic behaviour. The crystal orientation, as well as the elastic anisotropy, were also analysed and compared. First, crystal models with and without anisotropic elasticity were obtained to be used in contact problems. Second, a detailed study on a single asperity in contact with a rigid plane was carried out. Four cases were studied using the different constitutive laws. Thanks to these studies, the understanding of local deformation of an asperity, as well as variation induced by crystal plasticity models, were pointed out. Third, the study of the contact between a real measured rough surface and a rigid plane was carried out. This part was devoted, in one hand, to compare the same different behaviours as in the asperity study, and in a second hand to introduce the notion of contact tightness during the squashing of a rough surface. Thus, simple approach of fluid flow in the resulting free volume between two surfaces were analysed. In that way, we were able to show the impact of crystal plasticity on the contact transmissivity.

\section{REFERENCES}

[1] B.N.J. Persson, N. Prodanov, B.A. Krick, N. Rodriguez, N. Mulakaluri, W.G. Sawyer, and P. Mangiagalli. Elastic contact mechanics : Percolation of the contact area and fluid squeeze out. Eur. Phys. J. E, $35: 5: 1-17,2012$.

[2] F. Sahlin, R. Larsson, A. Almqvist, P.M. Lugt, and P. Marklund. A mixed lubrification model incorporating measured surface topography. part 1 : theory of flow factors. Proc. IMechE Part J : J. Engineering Tribology, 224, 2009.

[3] F. Sahlin, R. Larsson, P. Marklund, A. Almqvist, and P.M. Lugt. A mixed lubrification model incorporating measured surface topography. part 2 : roughness treatment, model validation, and simulation. Proc. IMechE Part J : J. Engineering Tribology, 224, 2009. 
[4] E.J. Abbott and F.A. Firestone. Specifying surface quality - a method based on accurate measurement and comparison. Mech. Eng., 55 :569, 1933.

[5] B. Bhushan. Surface roughness analysis and measurement techniques. Modern tribology handbook, 1 :49-119, 2001.

[6] J.A. Greenwood and J.B.P. Williamson. Contact of nominally flat surfaces. Proc. Royal Soc. London, A295 :300-319, 1966.

[7] D.J. Whitehouse and J.F. Archard. The properties of random surface of significance in their contact. Proc. Roy. Soc. Lond., 316 :97-121, 1970.

[8] A.W. Bush, R.D. Gibson, and T.R. Thomas. The elastic contact of a rough surface. Wear, $153: 53-64,1992$.

[9] A.W. Bush, R.D. Gibson, and G.P. Keogh. Strongly anisotropic rough surfaces. Journal of Lubrification Technology, 101 :15-20, 1979.

[10] A.W. Bush and R.D. Gibson. The elastic contact of a rough surface. Wear, 35 :87-111, 1987.

[11] W.R. Chang, I. Etsion, and D.B. Bogy. An elastic-plastic model for the contact of rough surfaces. J. of Tribology, 109 :257-263, 1987.

[12] Y. Zhao, D.M. Maietta, and L. Chang. An asperity microcontact model incorporating the transition from elastic deformations to fully plastic flow. J. of Tribology, 122 :86-93, 2000.

[13] Y. Zhao and L. Chang. A model of asperity interactions in elastic-plastic contact of rough surfaces. J. of Tribology, 123 :857-864, 2001.

[14] B. Bhushan. Contact mechanics of rough surfaces in tribology : multiple asperity contact. Tribology Letters, 4 :1-35, 1997.

[15] C.D. Yeo, R.R. Katta, J. Lee, and A.A. Polycarpou. Effect of asperity interactions on rough surface elastic contact behavior : Hard film on soft substrate. Tribology International, pages 1-42, 2010.

[16] L. Pei, S. Hyun, J.F. Molinari, and M.O. Robbins. Finite element modeling of elastoplastic contact between rough surfaces. Journal of the Mechanics and Physics of Solids, $53: 2385-2409,2005$.

[17] C. Vallet, D. Lasseux, P. Sainsot, and H. Zahouani. Real versus synthesized fractal surfaces : Contact mechanics and transport properties. Tribology International, $42: 250$ 259, 2009.

[18] C. Vallet, D. Lasseux, H. Zahouani, and P. Sainsot. Sampling effect on contact and transport properties between fractal surfaces. Tribology International, $42: 1132-1145$, 2009.

[19] B.N.J. Persson. Theory of rubber friction and contact mechanics. JOURNAL OF CHEMICAL PHYSICS, 115 :3840-3861, 2001.

[20] A. Majumdar and B. Bhushan. Fractal model of elastic-plastic contact between rough surface. J. of Tribology, $113: 1-11,1991$. 
[21] A. Majumdar and B. Bhushan. Elastic-plastic contact model of bifractal surfaces. Wear, $35: 87-111,1975$.

[22] V.A. Yastrebov, J. Durand, H. Proudhon, and G. Cailletaud. Rough surface contact analysis by means of the Finite Element Method and of a new reduced model. C. R. Mecanique, 339 :473-490, 2011.

[23] L. Méric, P. Poubanne, and G. Cailletaud. Single crystal modeling for structural calculations : Part 1 - model presentation. Journal of Engineering Materials and Technology, $113: 162-170,1991$.

[24] L. Méric and G. Cailletaud. Single crystal modeling for structural calculations : Part 2 - finite element implementation. Journal of Engineering Materials and Technology, $113: 171-182,1991$.

[25] E. Kröner. Zur plastischen verformung des vielkristalls. Acta Metall., $9: 155-161,1961$.

[26] R. Hill. Continuum micro-mechanisms of elastoplastic polycrystals. J. Mech. Phys. Sol., 13 :89-101, 1965.

[27] M. Berveiller and A. Zaoui. An extension of the self-consistent scheme to plastically flowing polycrystal. J. Mech. Phys. Sol., 26 :325-344, 1979.

[28] G. Cailletaud and P. Pilvin. Utilisation de modèles polycristallins pour le calcul par éléments finis. Revue européenne des éléments finis, 3 :515-541, 1994.

[29] G Martin, L. Nazé, and G. Cailletaud. Numerical multi-scale simulations of the mechanical behavior of beta-metastable titanium alloys Ti5553 and Ti17. Procedia Engineering, $10: 1803-1808,2011$.

[30] C. Vallet. Fuite liquide au travers d'un contact rugueux : application à l'étanchéité interne d'appareils de robinetterie. PhD thesis, Ecole Nationale Supérieure d'Arts et Métiers, 2008. 